\title{
Orbital period changes of the nova-like cataclysmic variable AC Cancri: evidence of magnetic braking and an unseen companion
}

\author{
S.-B. Qian ${ }^{1,2,3}$, Z.-B. Dai ${ }^{1,2,3}$, J.-J. He $e^{1,2,3}$, J. Z. Yuan ${ }^{1,2,3}$, F. Y. Xiang ${ }^{4}$, and M. Zejda ${ }^{5}$ \\ 1 National Astronomical Observatories/Yunnan Observatory, Chinese Academy of Sciences, PO Box 110, \\ 650011 Kunming, PR China \\ e-mail: qsb@ynao.ac.cn \\ 2 United Laboratory of Optical Astronomy, Chinese Academy of Science (ULOAC), 100012 Beijing, PR China \\ Graduate School of the CAS, Beijing, PR China \\ ${ }^{4}$ Physics Department, Xiangtan University, 411105 Xiangtan, Funan Province, PR China \\ e-mail: fyxiang@xtu.edu.cn \\ 5 Institute of Theoretical Physics and Astrophysics, Masaryk University, 61137 Brno, Czech Republic \\ e-mail: zejda@physics.muni.cz
}

Received 5 July 2006 / Accepted 27 October 2006

\section{ABSTRACT}

\begin{abstract}
Aims. The source AC Cnc is a nova-like cataclysmic variable containing a white-dwarf primary with a mass of $0.76 M_{\odot}$ and a K2-type secondary with a mass of $0.77 M_{\odot}$. We intend to study its period changes and search for evidence of magnetic braking and unseen third body.

Methods. The period changes were investigated based on the analysis of the $\mathrm{O}-\mathrm{C}$ curve, which is formed by one new eclipse time together with the others compiled from the literature.

Results. A cyclic change with a period of $16.2 \mathrm{yr}$ was found to be superimposed on a long-term period decrease at a rate of $\dot{P}=$ $-1.24( \pm 0.44) \times 10^{-8}$ days/year.

Conclusions. It is shown that the mechanism of magnetic activity-driven changes in the quadrupole momentum of the secondary star (Applegate's mechanism) does not explain it easily. This period oscillation was plausibly interpreted by a light-travel time effect caused by the presence of a cool M-type dwarf companion $\left(M_{3}>0.097 M_{\odot}\right)$ in a long orbit $(16.2 \mathrm{yr})$ around the binary. Since the masses of both components are nearly the same, the mass transfer from the lobe-filling secondary to the primary is not efficient to cause the continuous period decrease. It may be strong evidence of an enhanced magnetic stellar wind from the K2-type component. If the Alfén radius of the cool secondary is the same as that of the Sun (i.e., $R_{A}=15 R_{\odot}$ ), the mass-loss rate should be $\dot{M}_{2}=$ $-1.65 \times 10^{-10} M_{\odot} /$ year. By using the enhanced mass loss proposed by Tout \& Eggleton (1988), the mass-loss rate should be $\dot{M}_{2}=$ $-1.18 \times 10^{-9} M_{\odot} /$ year. In this case, the Alfén radius is determined to be $R_{A}=5.2 R_{\odot}$. However, the long-term decrease of the period may be only a part of a long-period ( $>100 \mathrm{yr}$ ) oscillation caused by the presence of an additional body. To check the conclusions, new precise times of light minimum will be required.
\end{abstract}

Key words. stars: novae, cataclysmic variables - stars: binaries: eclipsing - stars: individual: AC Cnc - stars: evolution stars: mass-loss - stars: binaries: close

\section{Introduction}

The evolution of long-period $(P>3 \mathrm{~h})$ cataclysmic variables $(\mathrm{CV})$ is governed by angular momentum (AM) loss via magnetic braking (Verbunt \& Zwaan 1981). However, the mechanism of magnetic braking is very complicated. It is driven by the magnetic field and stellar wind of the cool secondary component. When mass is driven off in the stellar wind, the ionized particles are forced to co-rotate with the magnetic field lines out to the Alfvén radius, which will remove AM from the secondary star and exert a braking force to slow it down. At the same time, the tidal lock of both components speeds it up and the AM is drawn from the binary orbit, causing it to shrink and the orbital period of the binary system to decrease. Therefore, the secular evolution of CVs driven by AM loss can in principle be discovered by measuring the variations in the orbital period of eclipsing CVs, and orbital period changes of eclipsing CVs are an observational probe of AM loss via magnetic braking. However, attempts to detect the long-term period decrease have been disappointing, since none of the analyzed stars presents a positive detection of an orbital period decrease (e.g., Beuermann \& Pakull 1984).

Nova-like CVs have never been observed to undergo nova or dwarf-nova-type outburst (Warner 1995), and predominantly have $P>3 \mathrm{~h}$, which implies a higher $\dot{M}_{2}$ and thus high AM loss (Hellier \& Naylor 1998). Therefore, this type of CVs is a more effective source for detecting AM loss. AC Cnc is a Nova-like CV consisting of a white dwarf and a K2-type dwarf. The eclipsing nature, the nearly same masses for both components, and the double-lined spectrum, all suggest that it is a very interesting system to study. In the paper, orbital period variations of AC Cnc were analyzed, then based on the properties of period changes, the triplicity, the magnetic braking, and the evolutionary states of this Nova-like CV were investigated. 
The light variability of AC Cnc was found by Kurochkin (1960), who classified it as an RR Lyr-type variable with a photographic magnitude range from 13.8 to $15 \mathrm{~m}$. . Kurochkin \& Shugarov (1980) later found it to be an eclipsing binary with a period of $7.2 \mathrm{~h}$. Based on UBV photometric observations, Shugarov (1981) subsequently suggested that AC Cnc is a novalike CV. Okazaki et al. (1982) discovered broad emission lines of $\mathrm{H}$ and $\mathrm{He}$ II superimposed on a continuum spectrum, which confirmed the CV nature of AC Cnc. Later, the CV nature of the object was also confirmed by the study of Yamasaki et al. (1983). Kurochkin \& Shugarov (1981) pointed out that AC Cnc could be identified with the X-ray souce H0850-13. Downes (1982) detected the spectrum of the secondary, which reveals it is a G- or K-type star. The first radial velocity curves for both components were obtained by Schlegel et al. (1984), who determined the absolute parameters of the system. However, the absolute parameters of AC Cnc derived by Schlegel et al. (1984) were obtained by using emission-line radial-velocity curves that exhibit phase shifts indicating that they could be unreliable. Timeresolved spectroscopic and photometric data of this nova-like CV were recently obtained by Thoroughgood et al. (2004) who determined reliable parameters of the system.

\section{Orbital period change of $A C$ Cancri}

Epochs and periods of the AC Cnc have been given by several authors (e.g., Kurochkin \& Shugarov 1980; Shugarov 1981; Kreiner et al. 2001; Thoroughgood et al. 2004). However, the binary system was neglected for period study. The CCD photometric observations of the binary system were carried out on March 25, 2006 with the PI1024 TKB CCD photometric system attached to the $1.0-\mathrm{m}$ reflecting telescope at the Yunnan Observatory in China. During the observation, the $R$ filter that is close to the Johnson's standard photometric system was used. A star near AC Cnc was chosen as the comparison. Those images were reduced by using PHOT (measure magnitudes for a list of stars) of the aperture photometry package of IRAF. With those observations, one CCD time of light minimum, HJD $2453821.1508 \pm 0.0006$, was derived by using a parabolic fitting method.

Times of light minimum of this binary were compiled by Thoroughgood et al. (2004) who obtained a linear ephemeris,

Min.I $=2451924.5368+0.30047747 \times E$.

After that, several new eclipse times were published by Dvorak (2005), Krajci (2006), and the present authors. All of the available times of light minimum of $\mathrm{AC} \mathrm{Cnc}$ are listed in the first column of Table 1 . The $(\mathrm{O}-\mathrm{C})_{1}$ values of those data from the linear ephemeris given by Kreiner et al. (2001),

Min.I $=2444290.3082+0.300477671 \times E$,

were computed and are shown in the fifth column of Table 1. Those listed in the third column of Table 1 are the observational methods where "pg" refers to photographic photometry, "Pe" to photoelectric, and "CCD" to charge-couple device photometry. The corresponding $(\mathrm{O}-\mathrm{C})_{1}$ curve is displayed Fig. 1 with photographic data, and the eclipse times observed with the methods of photoelectric (Pe) or CCD (hereafter "PC") photometry, respectively.

As shown in Fig. 1, the general $(\mathrm{O}-\mathrm{C})_{1}$ trend of $\mathrm{AC} \mathrm{Cnc}$ may reveal a continuous period decrease. Although those early photographic data show a slightly large scatter (up to 0.012), they should contribute to the general period change. By assigning a weight of 1 to the photographic observations and 5 to the PC data, a least-square solution yields

$$
\begin{aligned}
\text { Min.I }= & 2444290.30739( \pm 0.00091) \\
& +0.300477567( \pm 0.000000041) \times E \\
& -5.1( \pm 1.8) \times 10^{-12} \times E^{2}
\end{aligned}
$$

The quadratic term in Eq. (3) indicates a long-time period decrease at a rate of $\mathrm{d} P / \mathrm{d} t=-1.24( \pm 0.44) \times 10^{-8}$ days/year, which corresponds to a period increase of $0.107 \mathrm{~s}$ per century.

The $(\mathrm{O}-\mathrm{C})_{2}$ values from the quadratic ephemeris are displayed in Fig. 2. Although the $(\mathrm{O}-\mathrm{C})_{2}$ values of those photographic data show a large scatter, a cyclic change may be existed, which is mainly formed by those PC observations. A least-square solution based on all PC observations leads to

$$
\begin{aligned}
(\mathrm{O}-\mathrm{C})_{2}= & +0.0013( \pm 0.0011) \\
& +0.0026( \pm 0.0002) \sin [0.0183 \times E \\
& +124.5( \pm 3.6)]
\end{aligned}
$$

The sinusoidal term in Eq. (4) indicates a periodic change with a period of $P_{3}=16.2 \mathrm{yr}$ and an amplitude of $A_{3}=0.0026$. The residuals from Eq. (4) are listed in the seventh column of Table 1 and displayed in the lower panel of Fig. 3 where no changes can be traced. As shown in the upper panel of Fig. 3, the time of light minimum, HJD 50166.4481 given by Diethelm (1996), is important for constructing the periodic change. However, the error of the eclipse time is \pm 0.0005 indicating that this data point is reliable. The solid line in the low panel represents a linear fit to those residuals. The slope of this line is $+1.6 \times 10^{-8}$, which is much smaller than the error of the orbital period. As shown in Table 1, the residuals are no larger than 0.0009, and the value of the sum of the weighted squares of the residuals from Eq. (4) is $\Sigma=0.000054$ suggesting that Eq. (4) describes the general trend of the $(\mathrm{O}-\mathrm{C})_{2}$ curve well.

\section{Discussions-Mechanisms for orbital period variations}

\subsection{Applegate's mechanism}

As shown in Fig. 3, after the long-term period decreasing is subtracted from the $(\mathrm{O}-\mathrm{C})_{1}$ curve, the $(\mathrm{O}-\mathrm{C})_{2}$ residuals of all $\mathrm{PC}$ data suggest that there is a cyclic period variation with a period of $16.2 \mathrm{yr}$. AC Cnc is a nova-like cataclysmic variable consisting of a white dwarf primary and a K2-type secondary. One of the mechanisms that can explain that the periodic oscillation comes from magnetic activity-driven changes in the quadrupole momentum of the K2-type star, i.e., the so called Applegate's mechanism (e.g., Applegate 1992; Lanza et al. 1998). According to this mechanism, the redistributions of angular momentum within the $\mathrm{K} 2$ component could cause the changes in the quadrupole momentum and then result in the variations of the orbital period. Here, given the model proposed by Applegate (1992), we investigate the mechanism in detail.

The amplitude of the orbital period oscillation was calculated with

$\Delta P=\sqrt{2[1-\cos (2 \pi P / T)]} \times A$,

as $\Delta P=8.27 \times 10^{-7}$ days, where $P$ and $\Delta P$ are the orbital period and the amplitude of its variation, $A$ and $T$ are the amplitude and period of the $\mathrm{O}-\mathrm{C}$ oscillation, respectively. The absolute parameters of $\mathrm{AC} \mathrm{Cnc}$ determined by 
Table 1. All available times of light minimum for the nova-like cataclysmic variable AC Cancri.

\begin{tabular}{|c|c|c|c|c|c|c|c|}
\hline $\begin{array}{l}\text { JD Hel. } \\
2400000+\end{array}$ & Min. & Method & $E$ & $(\mathrm{O}-\mathrm{C})_{1}$ & $(\mathrm{O}-\mathrm{C})_{2}$ & Residuals & Ref. \\
\hline 34059.348 & I & $\mathrm{Pg}$ & -34049 & +0.0040 & +0.0072 & & (1) \\
\hline 36640.446 & I & $\mathrm{Pg}$ & -25459 & -0.0012 & +0.0003 & & (1) \\
\hline 36656.367 & I & $\mathrm{Pg}$ & -25406 & -0.0055 & -0.0040 & & (1) \\
\hline 37699.327 & I & $\mathrm{Pg}$ & -21935 & -0.0035 & -0.0025 & & (1) \\
\hline 41750.380 & I & $\mathrm{Pg}$ & -8453 & -0.0096 & -0.0093 & & (1) \\
\hline 42537.320 & I & $\mathrm{Pg}$ & -5834 & -0.0015 & -0.0011 & & (1) \\
\hline 42869.344 & I & $\mathrm{Pg}$ & -4729 & -0.0053 & -0.0049 & & (1) \\
\hline 43157.504 & I & $\mathrm{Pg}$ & -3770 & -0.0034 & -0.0029 & & (1) \\
\hline 43188.450 & I & $\mathrm{Pg}$ & -3667 & -0.0066 & -0.0061 & & (1) \\
\hline 43192.362 & I & $\mathrm{Pg}$ & -3654 & -0.0008 & -0.0003 & & (1) \\
\hline 43199.275 & I & $\mathrm{Pg}$ & -3631 & +0.0012 & +0.0017 & & (1) \\
\hline 43256.368 & I & Pg & -3441 & +0.0035 & +0.0040 & & (1) \\
\hline 43275.293 & I & $\mathrm{Pg}$ & -3378 & -0.0016 & -0.0011 & & (1) \\
\hline 43461.579 & I & Pg & -2758 & -0.0118 & -0.0112 & & (1) \\
\hline 43851.619 & I & Pg & -1460 & +0.0082 & +0.0089 & & (1) \\
\hline 43932.439 & I & Pg & -1191 & -0.0003 & +0.0004 & & (1) \\
\hline 43935.443 & I & $\mathrm{Pg}$ & -1181 & -0.0010 & -0.0003 & & (1) \\
\hline 43938.448 & I & $\mathrm{Pg}$ & -1171 & -0.0008 & -0.0001 & & (1) \\
\hline 43954.376 & I & Pg & -1118 & +0.0018 & -0.0011 & & (1) \\
\hline 44984.1119 & I & $\mathrm{Pe}$ & +2309 & +0.0008 & +0.0019 & -0.0000 & (2) \\
\hline 44985.3141 & I & $\mathrm{Pe}$ & +2313 & +0.0010 & +0.0021 & +0.0002 & (2) \\
\hline 44987.1165 & I & $\mathrm{Pe}$ & +2319 & +0.0006 & +0.0017 & -0.0002 & (2) \\
\hline 45457.6637 & I & $\mathrm{Pe}$ & +3885 & -0.0003 & +0.0010 & +0.0004 & (3) \\
\hline 46112.7032 & I & $\mathrm{Pe}$ & +6065 & -0.0021 & -0.0005 & +0.0004 & (4) \\
\hline 46114.8053 & I & $\mathrm{Pe}$ & +6072 & -0.0033 & -0.0017 & -0.0008 & (4) \\
\hline 46121.7177 & I & $\mathrm{Pe}$ & +6095 & -0.0019 & -0.0003 & +0.0007 & (4) \\
\hline 50166.4481 & I & CCD & +19556 & -0.0014 & +0.0034 & -0.0001 & (5) \\
\hline 51922.7339 & I & CCD & +25401 & -0.0076 & -0.0008 & -0.0001 & (6) \\
\hline 51923.6350 & I & CCD & 25404 & -0.0080 & -0.0012 & -0.0005 & (6) \\
\hline 51924.5367 & I & CCD & 25407 & -0.0077 & -0.0009 & -0.0002 & (6) \\
\hline 53329.8698 & I & CCD & 30084 & -0.0087 & -0.0001 & +0.0005 & (7) \\
\hline 53496.6341 & I & $\mathrm{Pe}$ & 30639 & -0.0095 & -0.0007 & -0.0004 & (8) \\
\hline 53821.1508 & I & CCD & 31719 & -0.0086 & +0.0007 & +0.0001 & (9) \\
\hline
\end{tabular}

References:

(1) Kurochkin \& Shugarov (1980); (2) Yamasaki et al. (1983); (3) Schlegel et al. (1984); (4) Zhang (1987); (5) Diethelm (1996); (6) Thoroughgood et al. (2004); (7) Dvorak (2005); (8) Krajci (2006); (9) The present.

Thoroughgood et al. (2004) are: $M_{1}=0.76 M_{\odot}, M_{2}=0.77 M_{\odot}$, $R_{2}=0.78 R_{\odot}$, and $a=2.21 R_{\odot}$. By using the equation (Applegate 1992; Lanza et al. 1998)

$$
\frac{\Delta P}{P}=-9\left(\frac{R_{2}}{a}\right)^{2} \frac{\Delta Q}{M_{2} R_{2}^{2}},
$$

the required variation in the quadruple moment $(\Delta Q)$ was computed as $\Delta Q=1.11 \times 10^{49} \mathrm{~g} \mathrm{~cm}^{2}$, in order to reproduce an orbital period change of $\Delta P=8.27 \times 10^{-7} \mathrm{~d}$.

During the computation, Applegate (1992) assumed the shell (the convective envelope) mass as $M_{\mathrm{s}}=0.1 M_{\odot}$; however, this is inappropriate for AC Cnc. As was done in the case of NN Ser by Brinkworth et al. (2006), we split the whole star into an inner core (described as 1) and an outer shell (described as 2). For each shell mass, the energy required to cause the observed period change was calculated and the results are shown in Fig. 4 where the required energy $\Delta E$ is plotted as a function of the mass of the outer shell $M_{\mathrm{s}}$ (Fig. 4). If we take the temperature of the K2-type component star as $T_{2}=5000 \mathrm{~K}$ (Cox 2000), its total radiant energy in the time interval of $16.2 \mathrm{yr}$ was computed as $E_{2}=7.60 \times 10^{41} \mathrm{erg}$. As we can see from the figure, when $M_{\mathrm{S}}<0.0561 M_{\odot}$, or $M_{\mathrm{S}}>0.5631 M_{\odot}, \Delta E$ is larger than $E_{2}$. A minimum value, $\Delta E=1.80 \times 10^{41} \mathrm{erg}$, was found when $M_{\mathrm{S}}=0.2347 M_{\odot}$. Thus, we could discover that Applegate's mechanism for the K2-type component may have difficulty for

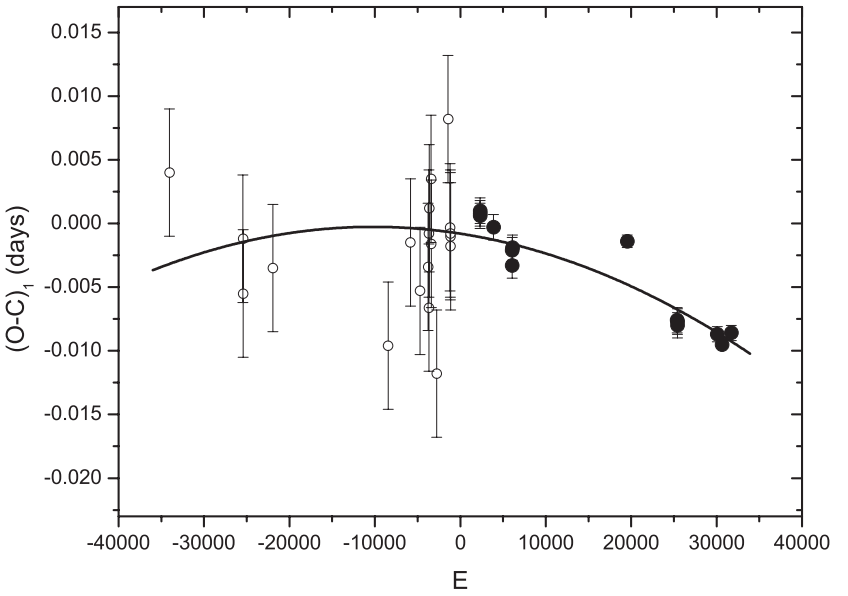

Fig. 1. $(\mathrm{O}-\mathrm{C})_{1}$ curve of AC Cancri respect to the linear ephemeris of Kreiner et al. (2000). Open circles refer to photographic observations and solid circles to photoelectric and CCD data. The solid line represents a long-term decreasing of the orbital period.

explaining the observed period oscillation, since the required minimum energy is too high when compared with its total radiant energy in the time interval of $16.2 \mathrm{yr}$, i.e., $\Delta E_{\text {minimum }} / E_{2}=$ $23.7 \%$. 


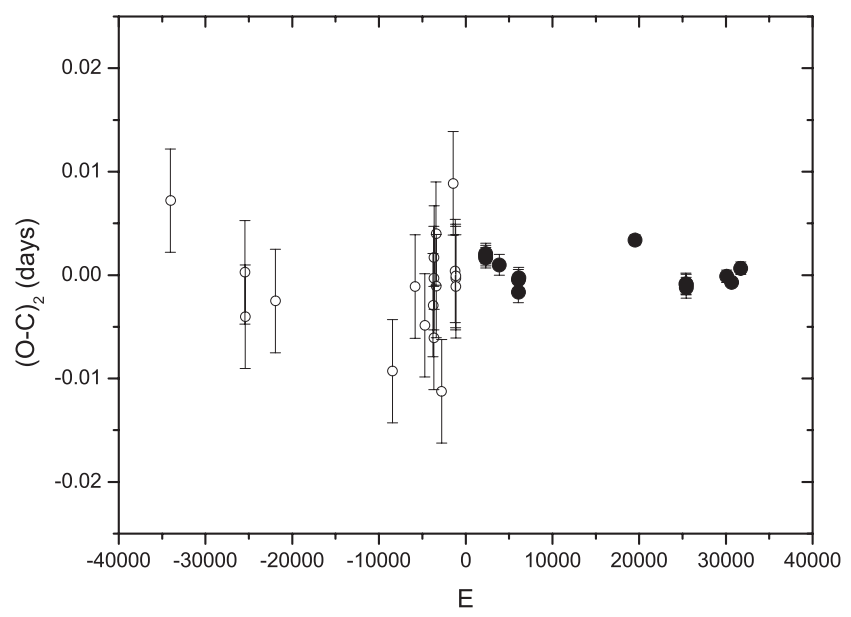

Fig. 2. $(\mathrm{O}-\mathrm{C})_{2}$ diagram of AC Cancri calculated with the quadratic ephemeris in Eq. (3). Symbols are the same as those in Fig. 1.

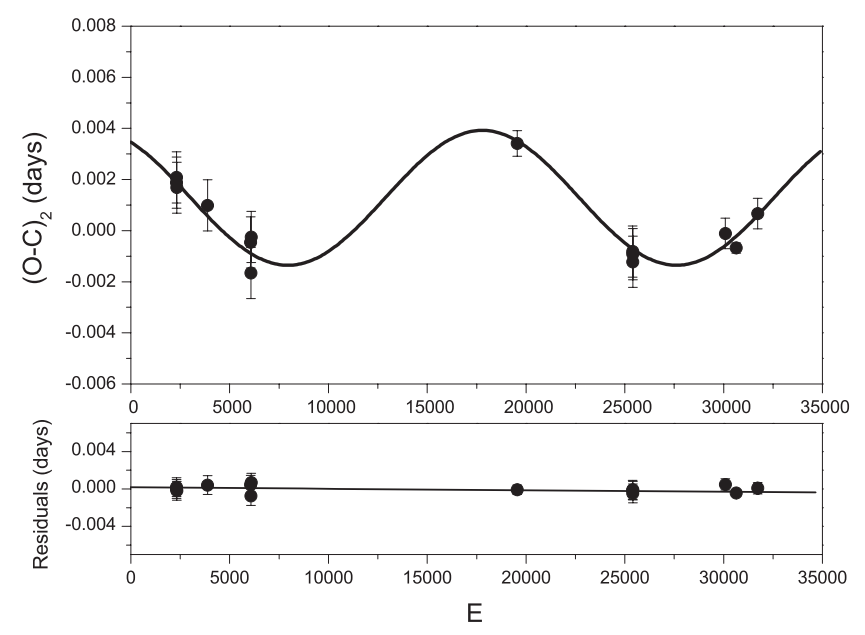

Fig. 3. $(\mathrm{O}-\mathrm{C})_{2}$ values of all photoelectric and $\mathrm{CCD}$ data for AC Cancri after the long-term period decreasing was removed. The solid line in the upper panel suggests that there is a small-amplitude cyclic oscillation in the orbital period. The residuals from the cyclic period change are displayed in the low panel.

\subsection{The presence of a tertiary component}

Since it may be difficult to use Applegate's mechanism to interpret the observed period oscillation of AC Cnc, a more plausible explanation of the periodic change in the orbital period is the light-travel time effect via the presence of a tertiary component (e.g., Borkovits \& Hegedüs 1996). As displayed in Fig. 3, the sine-like variation in the $\mathrm{O}-\mathrm{C}$ curve suggests that the eccentricity of the orbit of the tertiary component is close to zero. The projected radius of the orbit $a_{12}^{\prime} \sin i^{\prime}$ of the eclipsing pair rotating around the mass central of the triple system was computed with the equation

$a_{12}^{\prime} \sin i^{\prime}=A_{3} \times c$,

where $A_{3}$ is the amplitude of the $\mathrm{O}-\mathrm{C}$ oscillation and $c$ the speed of light. Then, a calculation with

$f(m)=\frac{4 \pi^{2}}{G P_{3}^{2}} \times\left(a_{12}^{\prime} \sin i^{\prime}\right)^{3}$,

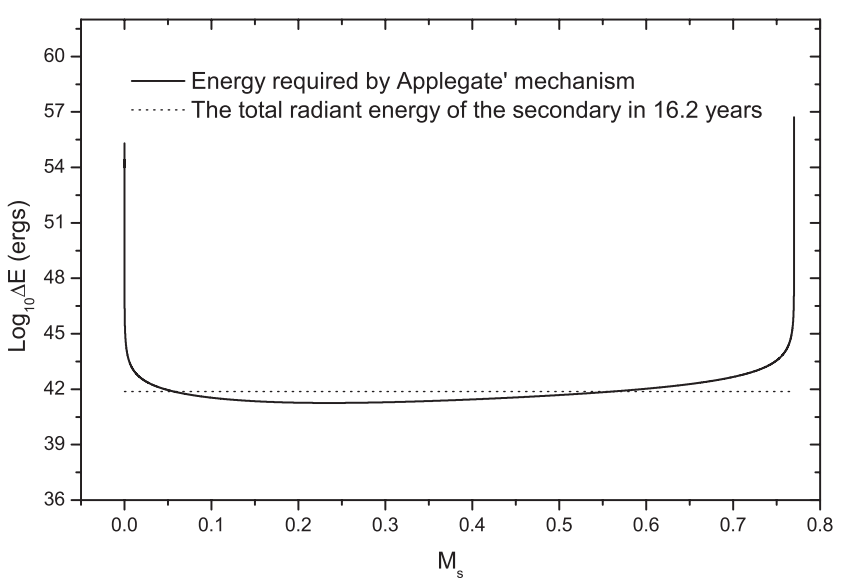

Fig. 4. A plot of the energy required to reproduce the period oscillation of AC Cancri by using Applegate's (1992) mechanism as a function of assumed shell mass of the secondary component (solid line). The dashed line is the total radiant energy of the secondary star in $16.2 \mathrm{yr}$.

where $G$ and $P_{3}$ are gravitational constant and the period of the $\mathrm{O}-\mathrm{C}$ oscillation, leads to a very small mass function: $f(m)=3.5( \pm 0.8) \times 10^{-4}$. The values of the masses and the orbital radii of the third component were estimated by using the equation

$f(m)=\frac{\left(M_{3} \sin i^{\prime}\right)^{3}}{\left(M_{1}+M_{2}+M_{3}\right)^{2}}$.

The relation between the orbital inclination and the mass of the tertiary component is plotted in Fig. 4. As shown in this figure, the tertiary component should be a cool M-type dwarf star, unless its orbital inclination is extremely low, i.e., $i^{\prime}<10^{\circ}$.

\subsection{Magnetic braking of the secondary component}

The general trend of the $(\mathrm{O}-\mathrm{C})_{1}$ curve displayed in Fig. 1 indicates that the period of AC Cnc is decreasing continuously at a rate of $\mathrm{d} P / \mathrm{d} t=-1.24( \pm 0.44) \times 10^{-8}$ days/year. Since the mass of the secondary $M_{2}$ is only slightly higher than that of the primary $M_{1}\left(M_{1}=0.76 M_{\odot}\right.$ and $M_{2}=0.77 M_{\odot}$, respectively $)$, the mass transfer from the lobe-filling secondary to the primary is not sufficient to cause the long-term period decrease. Tidal friction in a close binary can cause relatively rapid rotation, so that cool components in such a binary are presumably liable to stronger stellar wind than are single cool star. As a consequence, the binary can be subject to orbital angular momentum loss. Both the mass loss and the angular momentum loss may cause the orbital period reduction at a steady rate. Therefore, a plausible explanation to the period decrease in $\mathrm{AC} \mathrm{Cnc}$ is the magnetic braking of the K2-type component.

According to Tout \& Hall (1991), the relative rate of period change of a close binary containing a cool component is

$\frac{\dot{P}}{P}=-\frac{2 \dot{M}_{t}}{M}+\frac{3 \dot{M}\left(M_{2}-M_{1}\right)}{M_{1} M_{2}}+3 K$,

where $M_{1}, M_{2}$, and $M$ are the masses of the primary, the secondary, and the total mass; $\dot{M}_{t}$ is the mass loss rate from the whole system and $\dot{M}$ the rate of mass transfer between both components. The first term on the right-hand-side represents the stellar mass loss and can only cause an increase in period if $\dot{M}_{t}$ is negative. For AC Cnc, it can be ignored, because the mass loss 


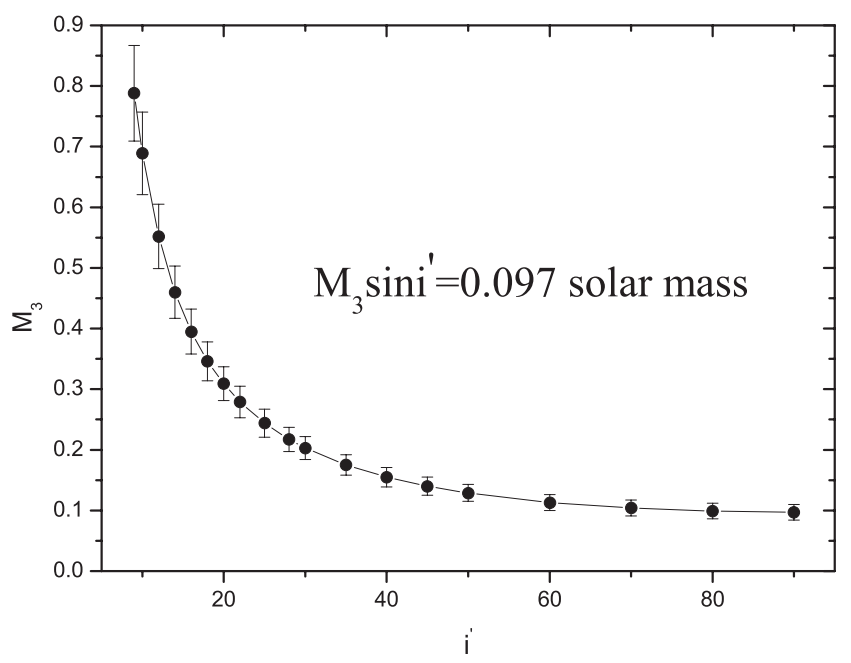

Fig. 5. The relation between the mass of the tertiary component $M_{3}$ and its orbital inclination $i^{\prime}$ in the nova-like cataclysmic variable AC Cancri.

from a low-mass binary is usually very small. The second term describes the mass-transfer contribution to the period change, and it can cause a decrease in period if the mass transfer is from the more massive component to the less massive one, but here it is also negligible because the mass of the secondary $M_{2}$ is close to that of the primary $M_{1}$. Finally, the third term indicates an additional angular momentum loss due to the co-rotation of an enhanced magnetic stellar wind, which may be the main cause of the period decreasing of $\mathrm{AC} \mathrm{Cnc.} \mathrm{It} \mathrm{is} \mathrm{given} \mathrm{by} \mathrm{the} \mathrm{equation}$ (Tout \& Hall 1991)

$K=\frac{2}{3}\left(\frac{R_{\mathrm{A}}}{a}\right)^{2} \frac{M}{M_{1} M_{2}} \dot{M}_{2}$

where $a$ and $R_{\mathrm{A}}$ are the distance between both components and the Alfvén radius, respectively. $\dot{M}_{2}$ refers to the mass loss from the K2 component. Therefore, Eqs. (10) and (11) can be combined to give

$\frac{\dot{P}}{P}=2\left(\frac{R_{\mathrm{A}}}{a}\right)^{2} \frac{M}{M_{1} M_{2}} \dot{M}_{2}$.

A calculation with Eq. (12) leads to: $R_{\mathrm{A}}^{2} \dot{M}_{2}=3.72 \times 10^{-8}$, in units of $R_{\odot}^{2} M_{\odot} /$ year. The corresponding relation between the Alfvén radius $R_{\mathrm{A}}$ and the mass-loss rate from the $K_{2}$ component is displayed in Fig. 6 in order to produce the observed period change by the magnetic braking.

By considering mass loss in an enhanced stellar wind, Tout \& Eggleton (1988) proposed

$\dot{M}_{2}=-4 \times 10^{-13} \frac{R_{2} L_{2}}{M_{2}}\left[1+10^{4}\left(\frac{R_{2}}{R_{L 2}}\right)^{6}\right]$.

For the nova-like cataclysmic variable AC Cancri, the K2 dwarf is filling the critical Roche Lobe, i.e., $R_{2}=R_{L 2}$. The massloss rate was computed as $\dot{M}=-1.38 \times 10^{-9} M_{\odot} /$ year. In this case, the required Alfvén radius should be $R_{\mathrm{A}}=5.2 R_{\odot}$ (Fig. 6). If the Alfén radius of the $\mathrm{K} 2$ dwarf secondary is the same as that of the Sun (i.e., $R_{\mathrm{A}} \sim 15 R_{\odot}$ ), its mass-loss rate should be $\dot{M}_{2}=-1.65 \times 10^{-10} M_{\odot} /$ year.

\subsection{Conclusions}

$\mathrm{AC} \mathrm{Cnc}$ is a nova-like $\mathrm{CV}$ containing a white dwarf and a K2-type main-sequence component. The masses of both

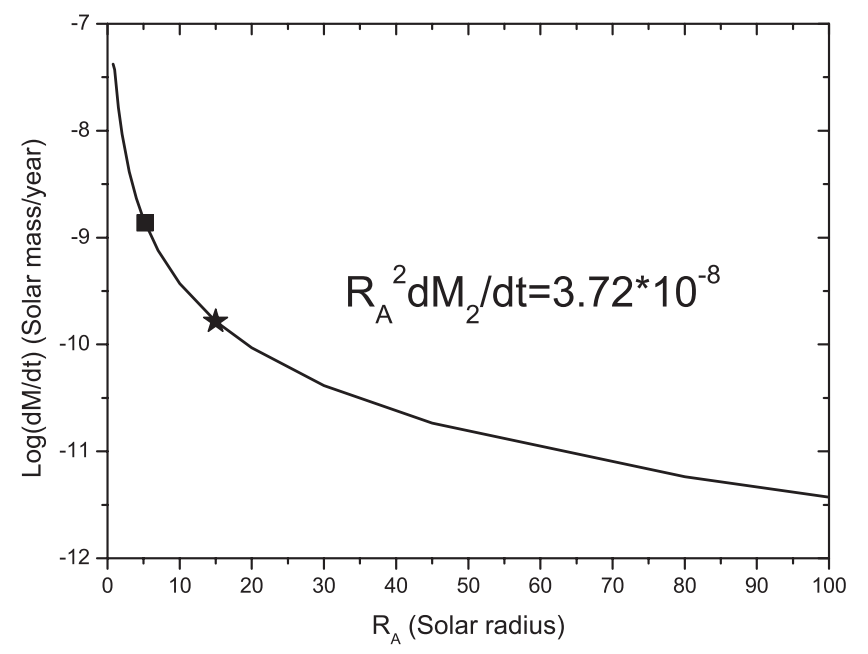

Fig. 6. The correlation between the Alfvén radius and the mass loss rate for the $\mathrm{K} 2$ dwarf of AC Cancri in order to cause the long-term period decreasing by magnetic braking. The solid star refers to a position where the same Alfvén radius as for the Sun was taken $\left(R_{A}=15 R_{\odot}\right)$, while the solid square represents the enhanced mass loss rate of Tout \& Eggleton (1988).

components are nearly the same. The changes in the orbital period of the eclipsing binary were analyzed based on one time of light minimum obtained with the 1.0-m telescope in Yunnan observatory, together with the others compiled from the literature. It was discovered that the period shows a cyclic change with a period of $16.2 \mathrm{yr}$, while it undergoes a long-term period decrease at a rate of $\dot{P}=-1.24( \pm 0.44) \times 10^{-8}$ days/year. Two physical mechanisms - Applegate's mechanism and the light-travel time effect via the presence of a tertiary component - are proposed to explain the period oscillation. It is shown that Applegate's mechanism may have difficulty causing the cyclic period variation because of the energy problem. The cyclic period oscillation may reveal the presence of a cool M-type dwarf in the system.

Since the masses of both components are nearly the same and the mass of the K2-type lobe-filling component $M_{2}$ is only slightly higher than that of the white dwarf component $M_{1}\left(M_{1}=\right.$ $0.76 M_{\odot}, M_{2}=0.77 M_{\odot}$ ), the mass transfer from the lobefilling secondary to the primary is not enough to cause the longterm period decrease. A plausible interpretation of the period decrease is the magnetic braking of the K2-type component. To produce the observed period decrease, the value of $R_{\mathrm{A}}^{2} \dot{M}$ should be $R_{\mathrm{A}}^{2} \dot{M}=4.02 \times 10^{-8}$. The corresponding relation between the Alfvén radius and the mass-loss rate from the $\mathrm{K} 2$ dwarf is displayed in Fig. 6. It is found that the observed period decrease can be explained by the enhanced stellar wind proposed by Tout \& Eggleton (1988). We have concluded that we may observe a genuine angular momentum loss via magnetic braking in the nova-like $\mathrm{CV}$, $\mathrm{AC} \mathrm{Cnc}$, which is thought to drive the long-term evolution of $\mathrm{CV}$ binary stars. In this case, the required Alfvén radius in the $\mathrm{K} 2$ dwarf star is $R_{\mathrm{A}}=5.2 R_{\odot}$. However, with the physical parameters derived by Thoroughgood et al. (2004), the angular momentum loss (AML) rate is determined to be $\dot{J}=-4.16 \times 10^{52} \mathrm{~g} \mathrm{~cm}^{2} \mathrm{~s}^{-2}$, which is more than an order of magnitude higher than the standard treatment of AML from magnetic braking in these systems (Rappaport et al. 1983) and six orders of magnitude higher than AML rates implied from open cluster data (e.g., Andronov et al. 2003). It is possible that the long-term period decrease is only a part of a long-period ( $>100 \mathrm{yr}$ ) oscillation caused by the presence of an additional 
body. In order to check this, new times are required for the light minimum in the future.

Acknowledgements. This work is partly supported by Yunnan Natural Science Foundation (No. 2005A0059M), Chinese Natural Science (No.10573032, No. 10573013 and No.10433030), and by Chinese Academy of Sciences (No.KJCXZ-SW-T06). CCD photometric observations of AC Cnc were obtained with the 1.0-m telescope at Yunnan Observatory. We thank the referee very much for the useful comments and suggestions that helped to improve this paper greatly.

\section{References}

Applegate, J. H. 1992, ApJ, 385, 621

Beuermann, K., \& Pakull, M. W. 1984, A\&A, 136, 250

Borkovits, T., \& Hegedues, T. 1996, A\&AS, 120, 63

Brinkworth, C. S., Marsh, T. R., Dhillon, V. S., \& Knigge, C. 2006, MNRAS, 365,287

Cox, A. N. 2000, Allen's Astrophysical Quantities, 4th edn. (New York: Springer), 392

Diethelm, R. 1996, BBSAG Bull. No. 111
Downes, R. A. 1982, PASP, 94, 950

Dvorak, S. W. 2005, IBVS No., 5603

Hellier, C., \& Naylor, T. 1998, MNRAS, 295, L50

Krajci, T. 2006, IBVS No. 5690

Kreiner, J. M., Kim, C.-H., \& Nha, I.-S. 2001, An Atlas of O-C Diagrams of Eclipsing Binaries, Krakóv.

Kurochkin, N. E. 1960, Astron. Tsirk., No. 212

Kurochkin, N. E., \& Shugarov, S. Yu. 1980, Astron. Tsirk., No. 1114

Lanza, A. F., Rodonò, M., \& Rosner, R. 1998, MNRAS, 296, 893

Okazaki, A., Kitamura, M., \& Yamasaki, A. 1982, PASP, 94, 162

Schlegel, E. M., Kaitchuck, R. H., \& Honeycutt, R. K. 1984, ApJ, 280, 235

Shugarov, S. Yu. 1981, Astron. Zh., 58, 583

Thoroughgood, T. D., Dhillon, V. S., Watson, C. A., et al. 2004, MNRAS, 353, 1135

Tout, C. A., \& Eggleton, P. P. 1988, MNRAS, 231, 823

Tout, C. A., \& Hall, D. S. 1991, MNRAS, 253, 9

Verbunt, F., \& Zwaan, C. 1981, A\&A, 100, 7

Warner, B. 1995, Cataclysmic Variable Stars (Cambridge: Cambridge Univ. Press)

Yamasaki, A., Okazaki, A., \& Kitamura, M. 1983, PASJ, 35, 423

Zhang, E. 1987, Acta Astrophys. Sinica, 7, 245 